\title{
A catalogue of Be stars in the direction of the Galactic Bulge ${ }^{\star}$
}

\author{
B. E. Sabogal ${ }^{1}$, R. E. Mennickent ${ }^{1}$, G. Pietrzyński ${ }^{1,2}$, J. A. García ${ }^{1}$, W. Gieren ${ }^{1}$, and Z. Kolaczkowski ${ }^{1}$ \\ 1 Universidad de Concepción, Departamento de Física, Casilla 160-C, Concepción, Chile \\ e-mail: bsabogal@udec.cl \\ 2 Warsaw University Observatory, Al. Ujazdowskie 4,00-478, Warsaw, Poland
}

Received 3 August 2007 / Accepted 8 October 2007

\begin{abstract}
Context. Detailed studies of Be stars in environments with different metallicities like the Magellanic Clouds or the Galactic Bulge are necessary to understand the formation and evolution mechanisms of the circumstellar disks. However, a detailed study of Be stars in the direction of the bulge of our own galaxy has not been performed until now.

Aims. The aim of this work is to report the first systematic search for Be star candidates in the direction of the Galactic Bulge. We present the full catalogue, give a brief description of the stellar variability seen, and show some light curve examples.

Methods. We searched for stars matching specific criteria of magnitude, color and variability in the $I$ band. Our search was conducted on the 48 OGLE II fields of the Galactic Bulge.

Results. This search has resulted in 29053 Be star candidates, 198 of them showing periodic light variations. Nearly 1500 stars in this final sample are almost certainly Be stars, providing an ideal sample for spectroscopic multi-object follow-up studies.
\end{abstract}

Key words. stars: emission-line, Be - Galaxy: bulge - catalogs

\section{Introduction}

Be stars are non-supergiant fast rotator B stars whose spectra have, or have had at some time, one or more Balmer lines in emission (Collins 1987). This emission originates from a flattened circumstellar disk and can come and go episodically on time scales of days to decades. The mechanisms responsible for the production and dynamics of the circumstellar gas are still not constrained. Possible mechanisms include non-radial pulsations, a wind-compressed disk model, magnetic activity and binarity (Porter \& Rivinius 2003, and references therein).

Be stars are variable in brightness on three time scales that are often superimposed. Many of them (especially early-type Be stars) show short-term photometric variability on time-scales of 0.2 to 2 days and amplitudes of up $0.1 \mathrm{mag}$, caused by non-radial pulsation or rotation (Percy et al. 2002, 2004). Some have midterm variations on times scales form weeks to months, probably due to density waves within the disk (Sterken et al. 1996). Their amplitudes extend up to $0.2 \mathrm{mag}$. They show also longterm variations from years to decades, with amplitudes of up 0.8 mag (Mennickent et al. 1994; Pavlovski et al. 1997; Hubert \& Floquet 1998; Percy \& Bakos 2001). Stagg (1987) found that this type of variability occurres in at least half of the Be stars. A few Be stars are close binaries and others present ejection processes due to magnetic activity, resulting in outbursts (Hubert et al. 1997).

Many Galactic Be stars have been surveyed for photometric variability in order to detect and confirm short-term or longterm variations and to find correlations between them and obtain clues to the physical processes in Be stars. For instance,

${ }^{\star}$ Full Tables 4 and 5 are only available in electronic form at the CDS via anonymous ftp to

cdsarc.u-strasbg.fr $(130.79 .128 .5)$ or via

http://cdsweb.u-strasbg.fr/cgi-bin/qcat?]/A+A/478/659
Hubert \& Floquet (1998) investigated the short-period variability of Be stars using an analysis based on the Fourier and CLEAN algorithms used on Hipparcos photometry. Percy et al. (2002, 2004) analyzed a large sample of stars with Hipparcos photometry using a form of an autocorrelation function. Typical problems in these studies are the gaps in the time distribution of the measurements and the limitations of the algorithms used.

In recent years, many Be star-like variables have been discovered in the Magellanic Clouds, showing a wide variety of light curves, some of them similar to those of the Galactic Be stars and others never observed in that type of star. Keller et al. (2002) concluded that most of these blue variables should be Be stars. Searches for Be stars in the Magellanic Clouds were performed by Mennickent et al. (2002), Keller et al. (1999, 2002) and Sabogal et al. (2005) on the basis of selection criteria applied to different photometric databases (OGLE II and $\mathrm{MACHO}$ ), and took into account the amplitude of the variability and ranges of color-magnitudes in the selection process. De Wit et al. (2006) investigated a subsample of the blue variables found by Mennickent et al. (2002) in the Small Magellanic Cloud and found that the photometric variability of these Be stars is due to variations in the amount of Bremsstrahlung due to the evolution of the circumstellar gas from a disk-shaped envelope towards a ring-like structure.

The study of Be stars is relevant for several important branches of stellar physics. In particular, detailed studies of Be stars in environments with different metallicities like the Magellanic Clouds or the Galactic bulge is crucial to understand the formation and evolution mechanisms of the circumstellar disks. However, a detailed study of Be stars in the direction of the bulge of our own galaxy has not been performed until now.

A very large number of stars was observed in the region of the Galactic Bulge during the second phase of the Optical Gravitational Lensing Experiment (OGLE II) 
(Udalski et al. 1997). We have performed a search for Be star candidates in this database. Here we present the results of this search.

\section{The data}

During the OGLE II project (Udalski et al. 1997, 2002), VI photometry maps of the Galactic center region were obtained. They contain astrometric and photometric data of about 30 million stars in 49 observed fields, and are already publicly available on the OGLE web page. The time base of these OGLE II observations was three years, and the 49 fields were monitored on all nights with good seeing conditions. The majority of observations were obtained using the $I$-band filter and a smaller number was obtained through the $V$-band filter (Udalski et al. 2002). From these maps we looked for stars with absolute $V$-band magnitudes in the typical range of the Galactic Be stars, i.e. $-6<M_{V}<0$ (Wegner 2000; Garmany \& Humphreys 1985). Assuming a distance modulus for the Galactic Bulge of 14.5 mag (Mc Namara et al. 2000), we obtained apparent magnitudes in the range of $8.5+A_{V}<V<14.5+A_{V}$, where $A_{V}$ is mean extinction value for each field of the Galactic Bulge obtained from Sumi (2004). As the saturation limit of the detector used by the OGLE II project is $12 \mathrm{mag}$ in the $V$-band our search was restricted to the range $12<V<14.5+A_{V}$ when the inferior limit $8.5+A_{V}$ was less than 12 mag.

We also constrained our search to the possible range of colours $V-I$ of classical Be stars. These colours are reddened due to the circumstellar disks and interstellar reddening (Wisniewski \& Bjorkman 2006), which is very strong in the direction of the galactic center. Therefore, we searched for stars with $-0.35<V-I<0.8+E(V-I)$, where $E(V-I)$ is the mean reddening value for each field of the Galactic Bulge obtained from Sumi (2004). He obtained extinction and reddening maps in the $V$ and $I$ bands for 48 OGLE II fields of the Galactic center region by using the average value of the ratio of total to selective extinction $R_{V I}=A_{V} / E(V-I)=1.964 \pm 0.085$. Because of the variable extinction in the direction of the Galactic center, these mean extinction and mean reddening values cover the following ranges: $0.676<E(V-I)<2.918 \mathrm{mag}$, and $1.327<A_{V}<5.733$ mag. Only in some fields do extinction and reddening reach high values. In particular, the OGLE II fields $B U L-S C 5, B U L-S C 37$ and $B U L-S C 43$ have the highest values of these parameters. Field $B U L-S C 44$ was not used in this search for Be star candidates because it was discarded by Sumi (2004) for his measurements of extinction and $E(V-I)$ and $A_{V}$ are not reported for this field.

When looking to the galactic bulge we observe many foreground and background stars. For this reason our selected sample might include stars whose distance moduli are different from that required by our selection criteria. In practice, this implies that the selected sample could be contaminated by foreground stars dimmer than normal Be stars and background stars brighter than normal Be stars.

Although OGLE II Galactic Bulge fields have high stellar densities and very strong and variable extinction, accurate photometry could be obtained from them (0.02-0.04 mag accuracy). However, $I$ magnitudes and $V-I$ colours for very red stars can be different from the standard values, reaching brighter $I$ magnitudes (up $0.25 \mathrm{mag}$ ) and redder $V-I$ values for stars with $(V-I)>2$ (Udalski et al. 2002).

Another important aspect of OGLE II Galactic Bulge fields is that due to crowding many stars can be blended, in particular those with I values close to 18 mag and fainter (Sumi et al. 2004).
Keeping in mind the above limitations, a total of 173404 stars were selected within the expected ranges of magnitudes and colours of Be stars at the Galactic Bulge distance. We have called them Be star precandidates. Table 1 shows for each field of the Galactic Bulge the total number of stars in the OGLE II database and the total number of stars with $V$ magnitudes and $V-I$ colours similar to those of Be stars. It also shows the number of selected Be star candidates and the number of the most certain Be star candidates, whose selection criteria are described in the following section. Finally this table shows the total number of stars for each column and its percentage with respect to the total sample of stars in the direction of the Galactic bulge in the OGLE II database.

\section{Results}

In order to obtain a list of certain Be star candidates in the direction of the bulge of our Galaxy, the following selection criteria were applied to the sample previously obtained (Be star precandidates).

\subsection{Selection criteria}

In order to determine the global variability properties of our sample and decide about the method of selection of Be star candidates, five fields: $B U L-S C 1, B U L-S C 10, B U L-S C 11, B U L-$ $S C 26$ and $B U L-S C 48$, far and near to the central region of the Galactic Bulge, were selected as test fields. Table 2 shows equatorial and galactic central coordinates of the selected fields. The I-band light curves for each previously selected star of the five fields were extracted from the OGLE II database.

Taking into account that large standard deviations of the I-band magnitudes are usually good indicators of stellar variability, a robust estimate of its mean magnitude $\overline{I_{R}}$ and standard deviation $\sigma_{R}$ was obtained for each star in the mentioned fields. This step was achieved by using a statistical C-program which is less sensitive to outliers than the usual ones (the C-code called SIGCOL is freely available at http://www.spaennare.se/ssphot.html. The reader can find at this page details about the method used by the program to calculate the robust estimate of each variable). Then the stars were grouped in $25 \mathrm{mag}$ bins and the average $\sigma_{R}$ and the average $\overline{I_{R}}$ were calculated per bin. In order to obtain a function $\Sigma\left(\overline{I_{R}}\right)$ that represents $\sigma_{R}$ as a function of $\overline{I_{R}}$, the data were fitted with a third-order polynomial. Stars with $\sigma_{R}$ values smaller than $\alpha \Sigma\left(\overline{I_{R}}\right)$ were rejected ( $\alpha$ is the selected threshold of variability). To select the $\alpha$ value, we plotted $\sigma_{R}$ against $\overline{I_{R}}$ for all stars in each field, and the obtained polynomial $\Sigma\left(\overline{I_{R}}\right)$. Figure 1 shows an example of this graph for field $B U L-S C 11$. The solid line represents the fitted polynomial. The distribution of the majority of stars is well fitted by this polynomial and the added function $\alpha \Sigma\left(\overline{I_{R}}\right)$ (dashed line) is a good limit between this distribution and that of possible variable stars. The best $\alpha$ value was 1.6 for for field $B U L-S C 1,1.5$ for $B U L-S C 10,1.6$ for $B U L-S C 11$, 1.7 for $B U L-S C 26$ and 2.0 for $B U L-S C 48$. For this reason we selected $\alpha=1.5$ as a conservative value for these five fields and used the same value for the remaining 43 fields.

In order to clean many light curves that showed sets of points much brighter or much fainter in magnitudes than those of the star (Fig. 2 presents the time series of two stars of BUL - SC39 field showing this problem) we constructed a pipeline that selects data between $\overline{I_{R}}-3 \sigma_{R}$ and $\overline{I_{R}}+3 \sigma_{R}$ for each star. Then the 
Table 1. Census of Be star precandidates and candidates in the direction of the Galactic Bulge.

\begin{tabular}{|c|c|c|c|c|}
\hline Field & $\begin{array}{l}\text { Total } \\
\text { of stars }\end{array}$ & $\begin{array}{l}\text { Total of Be star } \\
\text { precandidates }\end{array}$ & $\begin{array}{c}\text { Total of } \mathrm{Be} \\
\text { star candidates }\end{array}$ & $\begin{array}{c}\text { Total of most certain } \mathrm{Be} \\
\text { star candidates }\end{array}$ \\
\hline$B U L-S C 1$ & 729852 & 2407 & 273 & 30 \\
\hline$B U L-S C 2$ & 803269 & 2501 & 460 & 22 \\
\hline$B U L-S C 3$ & 805587 & 9694 & 1756 & 121 \\
\hline$B U L-S C 4$ & 774091 & 7449 & 1433 & 95 \\
\hline$B U L-S C 5$ & 433990 & 7306 & 1194 & 70 \\
\hline$B U L-S C 6$ & 514084 & 1055 & 209 & 10 \\
\hline$B U L-S C 7$ & 462748 & 1062 & 260 & 11 \\
\hline$B U L-S C 8$ & 401813 & 1833 & 358 & 8 \\
\hline$B U L-S C 9$ & 330338 & 1865 & 291 & 10 \\
\hline$B U L-S C 10$ & 458816 & 4112 & 320 & 31 \\
\hline$B U L-S C 11$ & 425984 & 3562 & 313 & 36 \\
\hline$B U L-S C 12$ & 534720 & 4282 & 700 & 40 \\
\hline$B U L-S C 13$ & 569850 & 3877 & 644 & 31 \\
\hline$B U L-S C 14$ & 619028 & 1735 & 274 & 10 \\
\hline$B U L-S C 15$ & 600787 & 1859 & 380 & 10 \\
\hline$B U L-S C 16$ & 699804 & 3053 & 599 & 27 \\
\hline$B U L-S C 17$ & 687019 & 2387 & 460 & 17 \\
\hline$B U L-S C 18$ & 749265 & 3511 & 599 & 21 \\
\hline$B U L-S C 19$ & 732089 & 3664 & 617 & 20 \\
\hline$B U L-S C 2 O$ & 785317 & 4566 & 847 & 20 \\
\hline$B U L-S C 21$ & 882518 & 3611 & 640 & 30 \\
\hline$B U L-S C 22$ & 715301 & 6434 & 1290 & 51 \\
\hline$B U L-S C 23$ & 723687 & 4697 & 890 & 48 \\
\hline$B U L-S C 24$ & 612189 & 2362 & 399 & 18 \\
\hline$B U L-S C 25$ & 622326 & 2224 & 397 & 15 \\
\hline$B U L-S C 26$ & 728200 & 1994 & 216 & 19 \\
\hline$B U L-S C 27$ & 690785 & 1890 & 280 & 11 \\
\hline$B U L-S C 28$ & 405799 & 1135 & 255 & 19 \\
\hline$B U L-S C 29$ & 491941 & 1098 & 176 & 7 \\
\hline$B U L-S C 30$ & 762481 & 3731 & 793 & 24 \\
\hline$B U L-S C 31$ & 790471 & 3673 & 590 & 28 \\
\hline$B U L-S C 32$ & 797493 & 2902 & 469 & 22 \\
\hline$B U L-S C 33$ & 738508 & 2502 & 566 & 25 \\
\hline$B U L-S C 34$ & 960656 & 4967 & 1144 & 57 \\
\hline$B U L-S C 35$ & 770940 & 3570 & 756 & 29 \\
\hline$B U L-S C 36$ & 873472 & 3005 & 561 & 37 \\
\hline$B U L-S C 37$ & 664424 & 10180 & 1740 & 80 \\
\hline$B U L-S C 38$ & 710234 & 2620 & 507 & 37 \\
\hline$B U L-S C 39$ & 584316 & 5897 & 1020 & 44 \\
\hline$B U L-S C 40$ & 630774 & 2795 & 493 & 24 \\
\hline$B U L-S C 41$ & 603404 & 2618 & 614 & 28 \\
\hline$B U L-S C 42$ & 600519 & 4367 & 1024 & 47 \\
\hline$B U L-S C 43$ & 474367 & 1406 & 242 & 17 \\
\hline$B U L-S C 45$ & 627412 & 2326 & 525 & 9 \\
\hline$B U L-S C 46$ & 551815 & 2078 & 374 & 27 \\
\hline$B U L-S C 47$ & 300705 & 3917 & 517 & 30 \\
\hline$B U L-S C 48$ & 286907 & 5137 & 352 & 41 \\
\hline$B U L-S C 49$ & 251629 & 2134 & 236 & 24 \\
\hline Total of stars & $\sim 30 \times 10^{6}$ & 173404 & 29053 & 1488 \\
\hline Percentage & $100 \%$ & $0.6 \%$ & $0.1 \%$ & $5 \times 10^{-3} \%$ \\
\hline
\end{tabular}

pipeline calculates amplitudes $A_{I}$ for the clean light curves, using the equation $A_{I}=\operatorname{Max} I-\min I$, where $\operatorname{Max} I$ and $\min I$ are the maximum and minimum values of the $I$ magnitudes. These amplitudes are always less than 1.00 mag. Finally, we select stars with amplitudes in the typical range of classical Be stars and also other types of blue variables like those found in the Magellanic Clouds (Mennickent et al. 2002). This step was achieved rejecting stars with amplitudes in the $I$-band of less than 0.05 mag and greater than $1.00 \mathrm{mag}$. As a test of the superior limit of 1.00 mag we calculated amplitudes for the uncleaned light curves of all stars in several fields, and visually inspected the light curves of those stars with amplitudes greater than 1.00. We found that all of them were similar to those of Fig. 2. About $30 \%$ of the light curves of all the fields showed photometric contamination like that shown in Fig. 2. The reason for this problem is being studied (Pietrzyński 2004).

Stars selected by the described process were called Be star candidates. Table 1 shows for each field of the Galactic Bulge the total number of Be star candidates.

To obtain a subsample of the most certain Be stars (i.e. those stars with similar light curves to those of classical Be stars and similar to those found in the Magellanic Clouds, Mennickent et al. 2002; Keller et al. 2002; Sabogal et al. 2005), we applied a statistical filter on the stellar $I$ magnitude distribution for each 


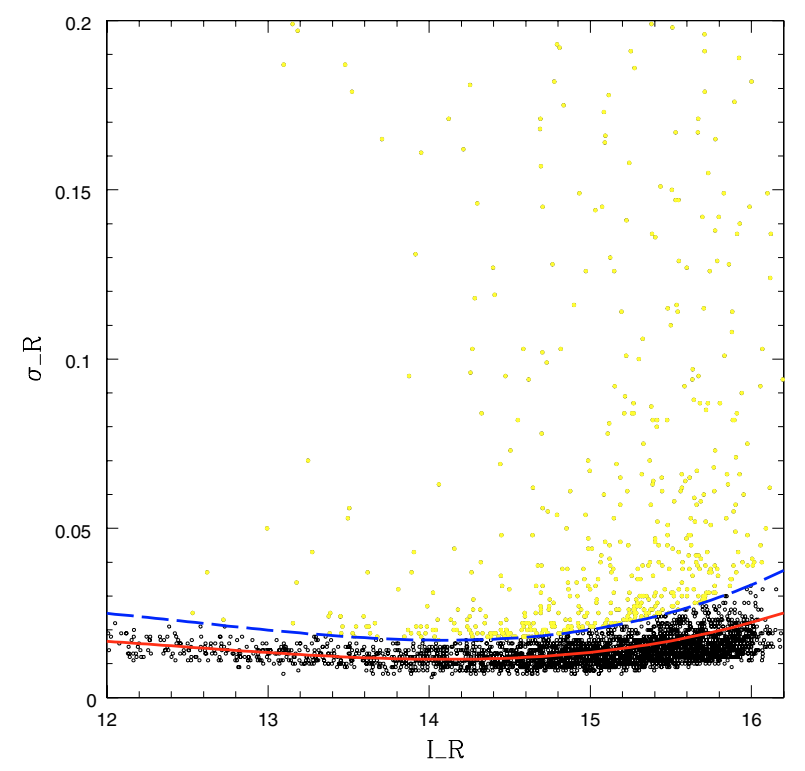

Fig. 1. Standar deviation $\sigma_{R}$ versus mean $I$-band magnitude $\overline{I_{R}}$ for all Be star precandidates in the $B U L-S C 11$ field. The solid line is the thirdorder polynomial $\Sigma\left(\overline{I_{R}}\right)$ fitted to the data as is explained in the text. The dashed line is the $\alpha \Sigma\left(\overline{I_{R}}\right)$ funtion, with $\alpha=1.5$.

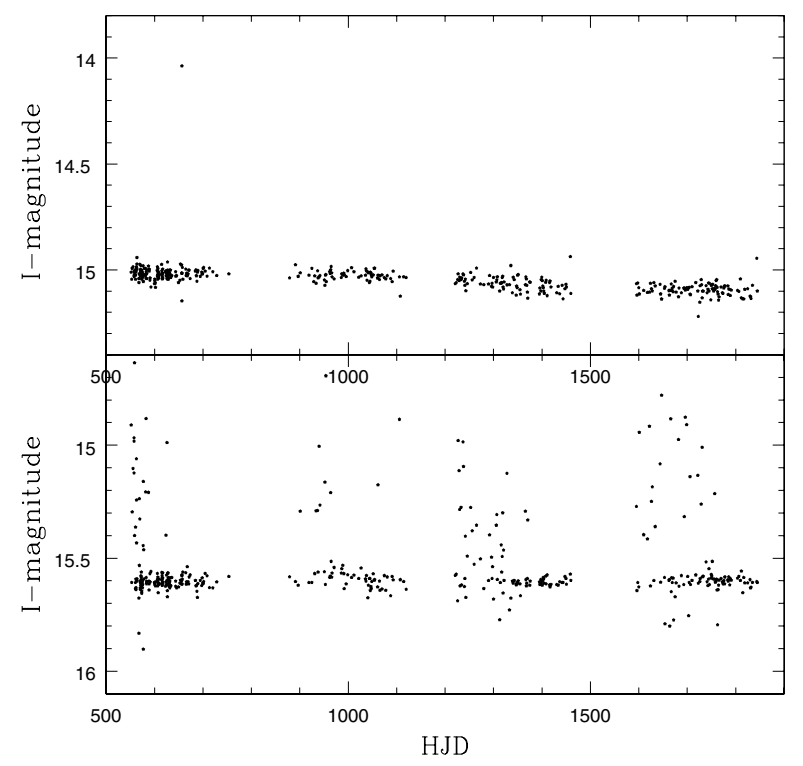

Fig. 2. Time series of $B U L-S C 39-127215$ (upper panel) and BULSC39- 743232 (botton panel) stars showing the photometric contamination observed in many light curves of the stars in the Galactic central region.

field. We first visually inspected the light curves of the stars in the five test fields mentioned before, and in field $B U L-S C 5$, and selected the most certain Be stars that we used as typical Bestar like variability indicators. We then searched for correlations between mean magnitude $(\bar{I})$, standard deviation $(\sigma)$, skewness $(S)$ and kurtosis $(K)$ of the magnitude distribution for each field, and the robust estimates for these quantities $\overline{I_{R}}, \sigma_{R}, S_{R}$ and $K_{R}$, respectively, where $S_{R}$ and $K_{R}$ are obtained by using the typical definitions of skewness $\left(S=\left(\sigma^{3}(N-1)\right)^{-1} \sum_{i=1}^{N}\left(I_{i}-\bar{I}\right)^{3}\right)$ and kurtosis $\left(K=\left(\sigma^{4}(N-1)\right)^{-1} \sum_{i=1}^{N}\left(I_{i}-\bar{I}\right)^{4}\right)$ but replacing $\bar{I}$ and $\sigma$ by $\overline{I_{R}}$ and $\sigma_{R}$. The aim of this step was to find a set of correlations between these parameters, whose ranges of values were
Table 2. Selected fields used to define our variability-based selection criteria. The epoch for the coordinates is year 2000 .

\begin{tabular}{ccccc}
\hline \hline Field & $\alpha$ (hh:mm:ss) & $\delta$ (dd:mm:ss) & $l\left(^{\circ}\right)$ & $b\left(^{\circ}\right)$ \\
\hline BUL-SC1 & $18: 02: 32.5$ & $29: 57: 41$ & 1.08 & 3.62 \\
BUL-SC10 & $18: 20: 06.6$ & $22: 23: 03$ & 9.64 & 3.44 \\
BUL - SC11 & $18: 21: 06.5$ & $22: 23: 05$ & 9,74 & 3.64 \\
BUL-SC26 & $17: 47: 15.5$ & $34: 59: 31$ & 4.90 & 3.37 \\
BUL-SC48 & $17: 28: 14.0$ & $39: 46: 58$ & 11.07 & 2.78 \\
\hline
\end{tabular}

Table 3. Parameters for the best correlations.

\begin{tabular}{ccc}
\hline \hline Name & Definition & Range of values \\
\hline$p_{1}$ & $\overline{I_{R}} / \bar{I}$ & {$[0.9970,1.0011]$} \\
$p_{2}$ & $\sigma_{R} / \sigma$ & {$[0.30,1.22]$} \\
$p_{3}$ & $S_{R} / S$ & {$[-1.00,7.15]$} \\
$p_{4}$ & $\left|p_{1} \times p_{2} \times p_{3}\right|$ & {$[0.29,3.82]$} \\
\hline
\end{tabular}

the same for all the magnitude distributions in all fields, in order to select the most certain Be stars without having to visually inspect thousands of light curves. To obtain these correlations we performed several plots of these statistical parameters and of aritmethic combinations of them (for example $K$ vs. $S, S_{R}$ vs. $S$, etc.) and selected only those showing very clear correlations between them. Due to the special shapes of the light curves of the most certain Be stars sample, we expected that skewness and kurtosis of the sample would be different to the reminding sample of Be star candidates, and could be useful to find correlations that would allow us to characterize the most certain $\mathrm{Be}$ star sample in order to select these stars without visual inspection. Indeed we found excellent correlations between $p_{1}=\overline{I_{R}} / \bar{I}$, $p_{2}=\sigma_{R} / \sigma, p_{3}=S_{R} / S$, and $p_{4}=\left|p_{1} \times p_{2} \times p_{3}\right|$. Parameters with the best correlations and their ranges of values are shown in Table 3. By using these correlations we could recover $70 \%$ of the previously most certain Be stars visually selected. This means that our method will eventually allow us to find a representative but not complete sample of the variable stars satisfying our color and magnitude criteria.

Then, we applied this filter to the total number of Be star candidates, which selects only the stars with magnitude distributions whose $p_{1}, p_{2}, p_{3}$ and $p_{4}$ values are in the ranges shown in Table 3 . We visually inspected the sample selected by this filter to obtain the subsample of most certain Be stars in the remaining fields. Table 1 shows for each field of the Galactic Bulge the total number of stars selected by this process.

The selection process used in this search for Be stars in the direction of the Galactic Bulge can be summarized in the following steps: first, we searched for stars with colours and magnitudes typical of Be stars. The new sample was called Be star precandidates. From these precandidates we then selected only those variable stars with amplitudes in the typical range of variability of Be stars of the Galaxy and the Magellanic Clouds. The obtained sample was called Be star candidates. Finally we used a statistical filter followed by visual inspection to select from the Be star candidates those with typical Be star-like variability. We called this final sample the most certain Be star candidates.

\subsection{Discussion of our sample of Be star candidates}

The final result of our search in the 48 OGLE II Galactic Bulge fields is a list of $29053 \mathrm{Be}$ star candidates and 1488 most certain Be star candidates that we provide as a catalog in the digital version of this article. Figure 3 shows some of the light curves of the 


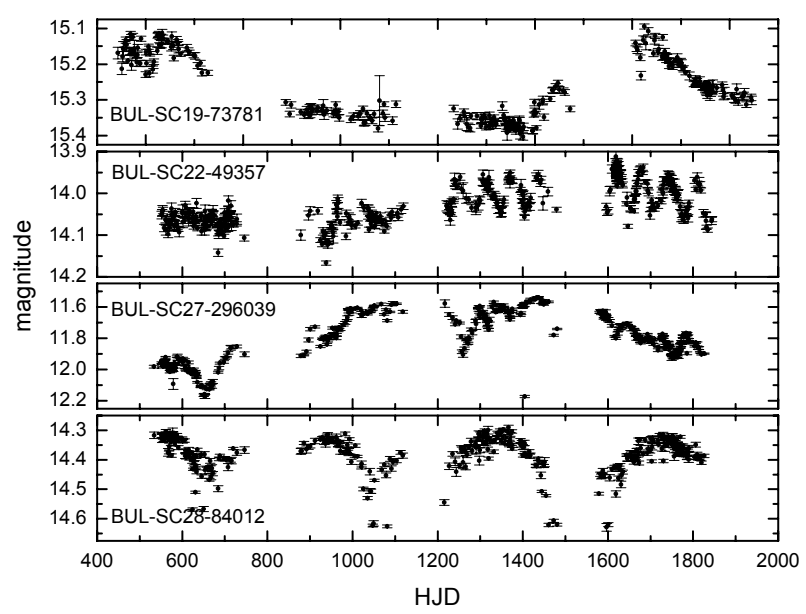

Fig. 3. Examples of light curves for the most certain Be star candidates in the direction of the Galactic Bulge.

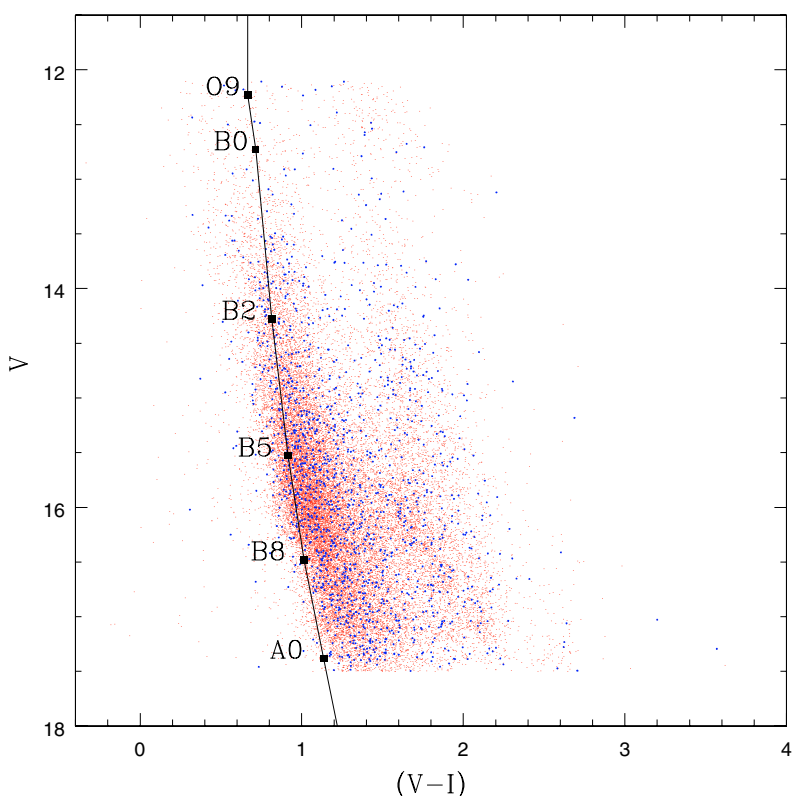

Fig. 4. $V$ vs. $(V-I)$ diagram for the selected Be star candidates. The track of the main sequence (MS) (Allen 2000) is shown for reference. Apparent $V$ magnitudes for it were calculated assuming the distance modulus of the Galactic bulge (14.5 mag) and $A_{V}=2.23$ (obtained by calculating the mean of $A_{V}$ values of the 48 Galactic Bulge fields). Reddened colours for the MS were obtained by using $E_{(V-I)}=1.135$, that is the mean of $E(V-I)$ values of the 48 Galactic Bulge fields.

most certain Be star candidates in the direction of the Galactic Bulge. The HJD zero point of the data is 2450000 . These light curves are typical of the selected sample, which has variable amplitudes, outbursts in some cases, small variations overlapping long-term variations, etc.

Table 4 contains the catalogue of the selected Be star candidates. This table gives the field, OGLE ID of the star, equatorial coordinates, $V$ magnitude, $V-I$ colour and $A_{I}$ amplitude (the complete table is available in electronic form). The first 1488 stars in this catalogue are those called the most certain Be star candidates. In Fig. 4 we show the $V$ versus $V-I$ diagram defined by the complete sample of stars. There is a clear sequence of many stars in the blue part of the diagram (where Be stars are expected) and the other sequence at the red part of
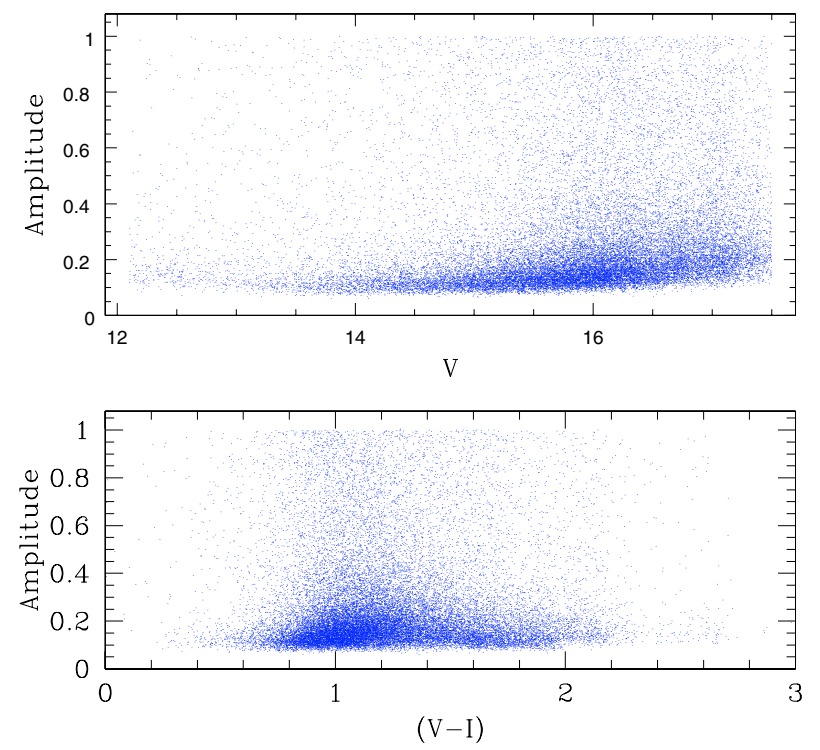

Fig. 5. $I$-amplitude vs. $V$ diagram (upper panel) and $I$-amplitude vs. $V-I$ diagram (bottom panel) for the selected Be star candidates

the diagram that contains fewer stars. Because of the reddening within each field of the Galactic bulge region, it is possible that many of these stars are actually Be stars, although we cannot discard the idea of a possible contamination by some red variables. Taking into account that the stellar population of the Galactic Bulge is dominated by old stars and that there is no evidence of massive objects, except in scattered regions and in the 1 parsec zone around the centre, where some stellar formation is detected (Rodgers \& Harding 1989; Bertelli et al. 1995; Martins et al. 2007), only some Be star candidates could belong to these regions of the Galactic Bulge. On the other hand, since the turnoff of the Galactic bulge main sequence is around $M_{V}=4$ (Reylé et al. 2004), and based on stellar proper motions and Galaxy models, it is known that the blue sequence observed in colourmagnitude diagrams of clusters and fields in the direction of the Galactic Bulge, and shown in Fig. 4, is due to the young stellar population of the disk (Zoccali et al. 2001; Udalski et al. 2002; Kuijken \& Rich 2002). Hence, we conclude that many of the Be star candidates in Fig. 4 probably belong to the Galactic disk.

Figure 5 shows the amplitude-magnitude (upper graph) and the amplitude-colour (bottom graph) diagrams for the Be star candidates, where amplitude values are those obtained by the process described in Sect. 3.1. It is observed from Fig. 5 that a large fraction of stars have amplitudes less than 0.2 magnitudes (the typical amplitude of Be stars). However, there are many stars with amplitudes larger than 0.2. Most of these stars have $V>15$ mag (see amplitude-magnitude diagram) and could be Be stars with long-term variability (Hubert et al. 1997) that have amplitudes up $0.8 \mathrm{mag}$. Some of them could be also Luminous Blue Variables, which show oscillations with amplitudes of half a magnitude (Humpreys \& Davidson 1994). We also observe in the amplitude-colour diagram that stars with amplitudes greater than 0.2 mag are mostly concentrated between $(V-I)=0.8$ and $(V-I)=1.8 \mathrm{mag}$. Based on these aspects and on the shapes of the light curves of the selected Be star candidates, it is possible that some of these stars are variables similar to those found in the Magellanic Clouds, classified as type- 1 to type 3 stars (Mennickent et al. 2002). 
Table 4. Catalogue of Be star candidates in the direction of the Galactic Bulge (complete table is available in electronic form at the CDS). The epoch for the coordinates is the year 2000 .

\begin{tabular}{ccccccc}
\hline \hline Field & ID & $\alpha$ (hh:mm:ss) & $\delta$ (dd:mm:ss) & $V$ & $V-I$ & $\mathbf{A}_{\mathbf{I}}$ \\
\hline BUL-SC1 & 116088 & 180204.67 & -294823.7 & 15.905 & 1.211 & 0.135 \\
$B U L-S C 1$ & 130042 & 180213.76 & -294518.8 & 15.285 & 1.05 & 0.118 \\
$B U L-S C 1$ & 213107 & 180216.96 & -301657.0 & 13.442 & 0.423 & 0.092 \\
$B U L-S C 1$ & 224746 & 180217.73 & -301431.8 & 14.604 & 0.919 & 0.118 \\
$B U L-S C 1$ & 257790 & 180230.79 & -300223.9 & 14.81 & 1.489 & 0.081 \\
$B U L-S C 1$ & 257829 & 180223.21 & -300403.0 & 15.144 & 1.067 & 0.112 \\
$B U L-S C 1$ & 291319 & 180218.64 & -295204.6 & 14.184 & 0.963 & 0.145 \\
$B U L-S C 1$ & 304035 & 180217.0 & -294904.0 & 14.275 & 0.861 & 0.104 \\
$B U L-S C 1$ & 304058 & 180230.7 & -294735.0 & 14.316 & 1.07 & 0.181 \\
$B U L-S C 1$ & 353800 & 180224.25 & -293550.6 & 15.093 & 0.816 & 0.129 \\
$B U L-S C 1$ & 36057 & 180203.41 & -301418.3 & 14.634 & 1.368 & 0.129 \\
$B U L-S C 1$ & 365772 & 180223.09 & -293219.3 & 15.502 & 0.938 & 0.130 \\
$B U L-S C 1$ & 376373 & 180240.19 & -302412.4 & 13.999 & 0.738 & 0.316 \\
$B U L-S C 1$ & 376671 & 180246.84 & -302342.2 & 15.813 & 1.046 & 0.176 \\
$B U L-S C 1$ & 420820 & 180235.11 & -301005.8 & 15.328 & 0.855 & 0.103 \\
$B U L-S C 1$ & 441953 & 180243.56 & -300328.5 & 14.995 & 0.931 & 0.115 \\
$B U L-S C 1$ & 452151 & 180234.63 & -295951.0 & 15.606 & 0.993 & 0.118 \\
$B U L-S C 1$ & 462569 & 180238.07 & -295510.4 & 14.002 & 0.868 & 0.097 \\
$B U L-S C 1$ & 523070 & 180238.2 & -293858.9 & 13.454 & 1.307 & 0.406 \\
\hline
\end{tabular}
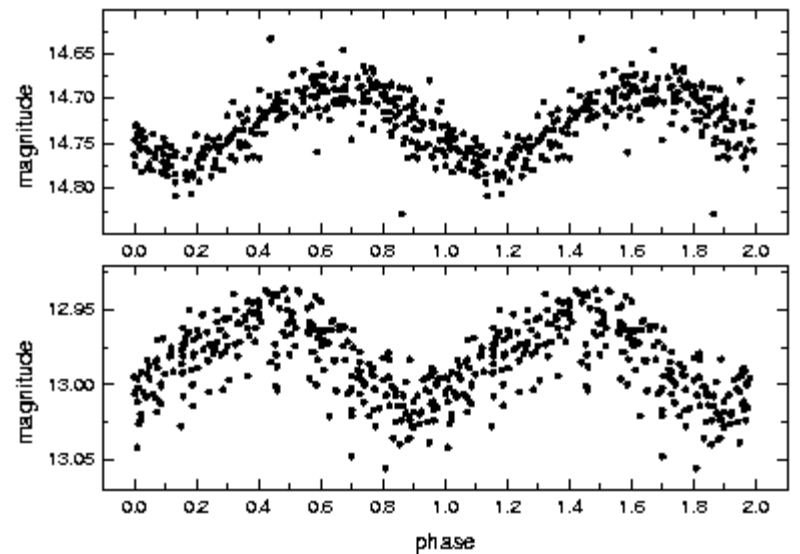

Fig. 6. Example of light curves for two periodic variables: $B U L-S C 13-$ 204056 with $P=7.06$ days (upper panel) and BUL - SC13 - 204056, with $P=4.64$ days (bottom panel).

\subsubsection{Periodic Be star candidates}

In order to investigate whether some of the Be star candidates show short-term, mid-term or long-term variations, we searched for periodicities in their light curves. The duration and frecuency of observations of the OGLE II project were enough to detect mid an long-term variability timescales, and also those shortterm variations with periods greater than 1 day.

For this search we used the analysis of variances algorithm (AOV) (Schwarzenberg-Czerny 1989). We then used special software provided by one of the authors (Piertzyński 2004) to graph phased light curves and confirm or reject the obtained periods. We discarded false periods and eclipsing binaries. Finally, only 198 Be star candidates (0.68 percent) turned out to be periodic variables. Table 5 presents a list of names, periods and errors of these stars (complete table is available in electronic form at the CDS). These errors were calculated by measuring the half width of the frequency peak in the power spectrum. Figure 6 shows phased light curves of two of the periodic variables found. These curves and many of the phased light curves of the periodic variables found closely resemble

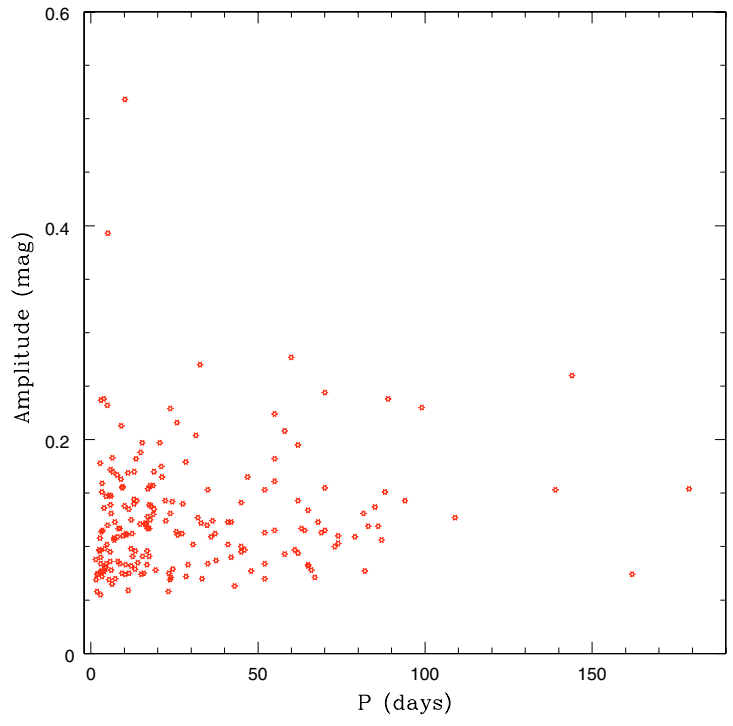

Fig. 7. Amplitude vs. period diagram for the periodic Be star candidates.

sinusoids indicating that these stars could be binaries. Some of these periodic Be star candidates show several periods in the periodogram. On the basis of this fact these stars could be multiperiodic variables similar to those A and B emission-line stars studied by Mennickent et al. (2006) in the analysis of a subsample of bright type-3 stars of the SMC. We will perform a detailed study of this sample of periodic stars in a future work. For the moment we give in Table 5 only the fundamental period obtained for each star.

Figure 7 shows the period-amplitude diagram for the 198 periodic Be star candidates. These amplitudes were obtained by subtracting the maximum and minimum value of each light curve. These values were given by the software used to graph light curves and confirm or reject the obtained periods.

It is shown in Fig. 7 that all these stars have periods greater than 1 day. This allows us to discard the possibility that some of these stars could be $\beta$ Cepheid stars whose periods are only several hours (Stankov \& Handler 2005). It is also observed in the diagram that stars have amplitudes greater than 0.06 mag, 
Table 5. List of periodic Be star candidates in the direction of the Galactic Bulge (complete table is available in electronic form at the CDS).

\begin{tabular}{cccc}
\hline \hline Field & ID & Period (days) & Error (days) \\
\hline$B U L-S C 1$ & 365679 & 21.3 & 0.3 \\
$B U L-S C 1$ & 628586 & 1.574 & 0.002 \\
$B U L-S C 2$ & 178538 & 65 & 3 \\
$B U L-S C 2$ & 266251 & 70 & 5 \\
$B U L-S C 2$ & 517884 & 35 & 1 \\
$B U L-S C 3$ & 202194 & 17.8 & 0.2 \\
$B U L-S C 3$ & 328070 & 10.12 & 0.05 \\
$B U L-S C 3$ & 355285 & 9.0 & 0.4 \\
$B U L-S C 3$ & 355797 & 12.8 & 0.2 \\
$B U L-S C 3$ & 444397 & 25.8 & 0.3 \\
$B U L-S C 3$ & 455478 & 63 & 6 \\
$B U L-S C 3$ & 501384 & 55 & 8 \\
$B U L-S C 3$ & 525314 & 55 & 2 \\
$B U L-S C 3$ & 646913 & 13.5 & 0.2 \\
$B U L-S C 3$ & 657652 & 6.1 & 0.1 \\
$B U L-S C 3$ & 681277 & 3.94 & 0.01 \\
$B U L-S C 4$ & 120636 & 33 & 3 \\
$B U L-S C 4$ & 144329 & 23.8 & 0.6 \\
$B U L-S C 4$ & 155897 & 24.4 & 0.6 \\
\hline
\end{tabular}
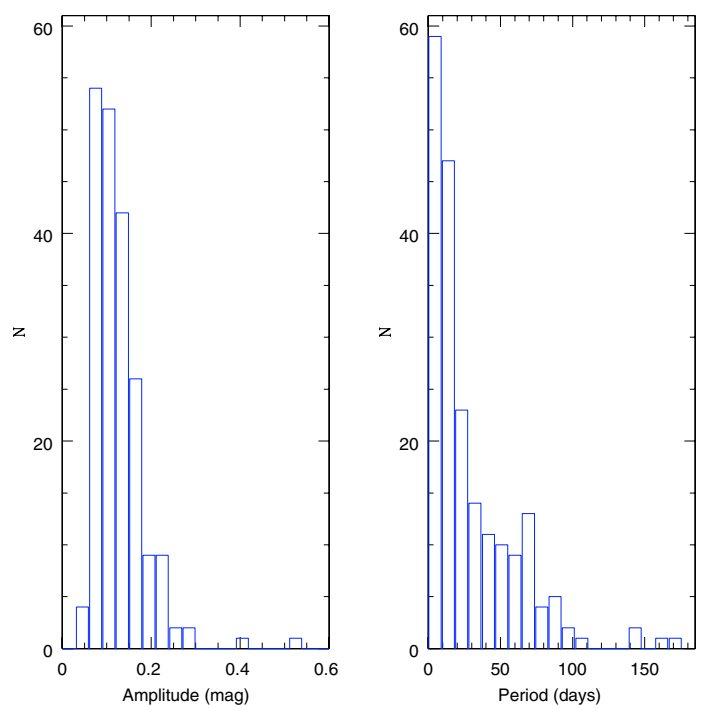

Fig. 8. Amplitude and period histogram for the periodic Be star candidates.

rejecting the idea that they could be Slow Pulsating B stars which have amplitudes of several milimagnitudes. This was expected because of the selection criteria of our search, which rejected stars with amplitudes of less than $0.05 \mathrm{mag}$. The diagram in Fig. 7 shows the largest concentration of stars located in a range of periods between 1 and 20 days, and amplitudes between 0.06 and $0.2 \mathrm{mag}$. This is confirmed in the period and amplitude histograms shown in Fig. 8 (right and left panels, respectively). It shows that 30 percent of the periodic variables have period lesser than 10 days and amplitudes lesser than $0.1 \mathrm{mag}$. This result is similar to that found for $13 \mathrm{Be}$ stars in the Small Magellanic Cloud, which present short-term photometric variations with periods less than 2.5 days and amplitudes less than 0.1, superimposed on long-term variations (Martayan et al. 2007). The amplitude histogram also shows that 92 percent of the sample has amplitudes of less than $0.2 \mathrm{mag}$, and only 8 percent of them have amplitudes greater than 0.2 mag. These ranges of periods and amplitudes are comparable to those of Galactic Be stars with short and mid-term photometric variability.

\section{Conclusion}

In this paper we have provided a catalogue of 29053 Be star candidates, 198 of them periodic, in the direction of the Galactic Bulge that were selected on photometric criteria. Most of these Be star candidates are probably members of the Galactic disk and trace the gradient of metallicity towards the Galactic centre. They are ideal targets for future observing programs based on multi-object spectroscopy, narrow band photometry or $\mathrm{H} \alpha$ imaging surveys. These programs could eventually establish their true nature and breake the residual degeneracy with variable red giants in the red part of the $(V-I)$ color distribution.

Acknowledgements. We thank the referee, Dr Willem Jan de Wit, for his valuable suggestions and comments that improved this work. B.S. and A.G. acknowledge financial support for this work from Programa MECESUP de Estadías de Estudiantes Tesistas en Centros de Excelencia en el Extranjero USA0108, and B.S. acknowledges support by Programa de Becas de Doctorado MECESUP UCO0209. R.E.M. acknowledges support by Fondecyt grant 1070705. W.G., G.P. and R.E.M. acknowledge financial support for this work from the Chilean Center for Astrophysics FONDAP 15010003.

\section{References}

Allen, C. W. 2000, in Allen's Astrophysical Quantities, forth edition, ed. A. Cox (New York: AIP Springer-Verlag).

Bertelli, G., Bressan, A., Chiosi C., Ng, Y. K., \& Ortolani S. 1995, A\&A, 301, 381

Collins, G. W. 1987, in Physics of Be stars, Proceedings of the Ninety-second IAU Colloquium (Boulder, CO. Cambridge and New York, Cambridge University Press)

De Wit, W. J., Beaulieu, J. P., Lamers, H. J. G. L. M., Lesquoy, E., \& Marquette, J. B. 2003, A\&A 410, 199

De Wit, W. J., Lamers, H. J. G. L. M., Marquette, J. B., \& Beaulieu, J. P. 2006, A\&A, 456, 1027

Garmany, C. D., \& Humphreys, R. M. 1985, AJ, 90, 2009

Hubert, A. M., \& Floquet, M. 1998, A\&A 335, 565

Hubert, A. M., Floquet, M., Gomez, A. E., \& Aletti, V. 1997, Proceedings of the ESA Symposium 'Hipparcos - Venice '97', ESA SP-402, 315

Humphreys, R. M., \& Davidson, K. 1994, PASP, 106, 1025

Keller, S. C., Wood, P. R., \& Bessell, M. S. 1999, A\&AS, 134, 489

Keller, S. C., Bessell, M. S., Cook, K. H., Geha, M., \& Syphers, D. 2002, AJ, 124,2039

Kuijken, K., \& Rich, R. M. 2002, ApJ, 124, 2054

Martayan, C., Floquet, M., Hubert, A., \& Mekkas, M. 2007, PASPCS, 124, 460

Martins, F., Genzel R., Hillier, D. J., et al. 2007, A\&A, 468, 233

McNamara, D. H., Madsen, J. B., Barnes, J., \& Ericksen, B. F. 2000, PASP, 112,202

Mennickent, R. E., Vogt, N., \& Sterken, C. 1994, A\&AS, 108, 237

Mennickent, R. E., Pietrzynski, G., Gieren, W., \& Szewczyk O. 2002, A\&A, 393,887

Mennickent, R. E., Cidale, L., Pietrzyński, G., Gieren, W., \& Sabogal, B. 2006, A\&A, 457, 949

Pavlovski, K., Harmanec, P., Bozić, H., et al. 1997, A\&AS, 125, 75

Percy, J. R., \& Bakos, A. G. 2001, PASP, 113, 748

Percy, J. R., Hosick, J., Kincaide, H., \& Pang, C. 2002, PASP, 114, 551

Percy, J. R., Harlow, C. D. W., \& Wu, A. P. S. 2004, PASP, 116, 178

Pietrzyński, G. 2004, private communication

Porter, J. M., \& Rivinius, T. 2003, PASP, 115, 1153

Reylé, C., Robin, A. C., Schultheis, M., \& Picaud, S. 2004, Proceedings of the Gaia Symposium The Three-Dimensional Universe with Gaia, ESA SP-576, ed. C. Turon, K. S. O'Flaherty, \& M. A. C. Perryman

Rodgers, A. W., \& Harding, P. A. 1989, AJ, 97, 1036

Sabogal, B. E., Mennickent, R. E., Pietrzyński, G., \& Gieren, W. 2005, MNRAS, 361,1055

Schwarzenberg-Czerny. 1989, MNRAS, 241, 153

Stankov, A., \& Handler, G. 2005, ApJS, 158, 193

Sterken, C., Vogt, N., \& Mennickent, R. E. 1996, A\&A, 311, 579

Sumi, T. 2004, MNRAS, 349, 193

Sumi, T., Wu, X., Udalski, A., et al. 2004, MNRAS, 348, 1439

Udalski, A., Kubiak, M., \& Szymanski, M. 1997, Acta Astron., 47, 319

Udalski, A., Szymanski, M., Kubiak, M. et al. 2002, Acta Astron., 52, 217

Wegner, W. 2000, MNRAS, 319, 771

Wisniewski, J. P., \& Bjorkman, K. S. 2006, ApJ, 652, 458

Zoccali, M., Renzini, A., Ortolani, S., Bica, E., \& Barbuy, B. 2001, AJ, 121, 2638 